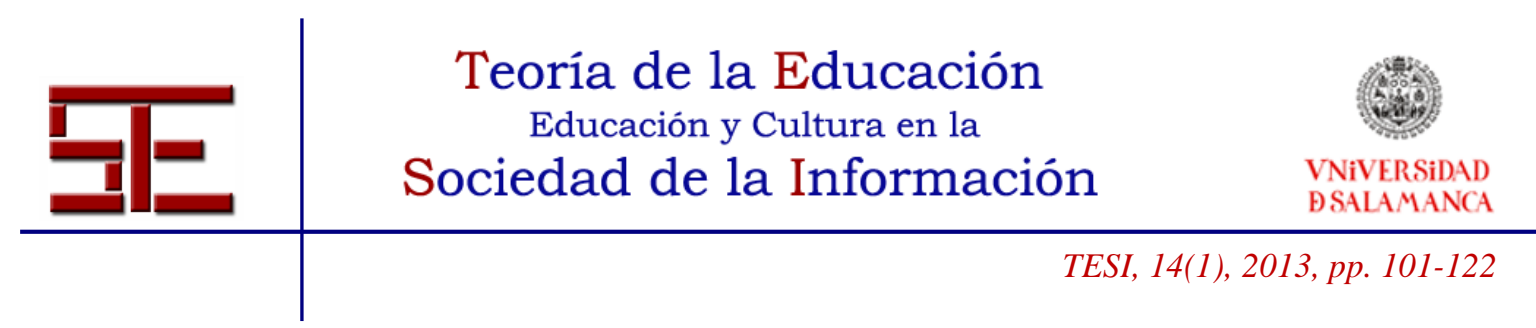

\title{
ANÁlisis DEL ENTORNO COLABORATIVO CREADO PARA UNA EXPERIENCIA DE MOBILE LEARNING**
}

Resumen: Se presentan los resultados de una investigación sobre las concepciones y usos de dispositivos móviles de un grupo de estudiantes que trabajaron en un entorno de aprendizaje colaborativo como parte un proceso de e-learning. Se emplearon dos métodos de investigación, en primer lugar el análisis del contenido de los mensajes de un foro de discusión enviados durante todo el curso, para recoger datos acerca del uso y la valoración que hacían del proceso de mobile learning. Posteriormente, mediante las entrevistas en profundidad, se analizó la percepción de mobile learning y los cambios producido durante el curso en el entorno colaborativo de aprendizaje. Los resultados indican que el entorno de intercambio creado amplifica la participación y colaboración entre los alumnos en el proceso de mobile learning, favoreciendo un mayor protagonismo de los estudiantes en una experiencia de aprendizaje online.

Palabras clave: foro de debate; aprendizaje online; entornos colaborativos; mobile learning; entrevista.

* Esta investigación se lleva a cabo con el soporte del Departamento de Innovación del Institute for LifeLong Learning IL3 of University of Barcelona, y cuenta con la participación de los alumnos del "Posgrado en Community Manager \& Social Media". Se agradece el soporte del departamento y la participación de los alumnos.

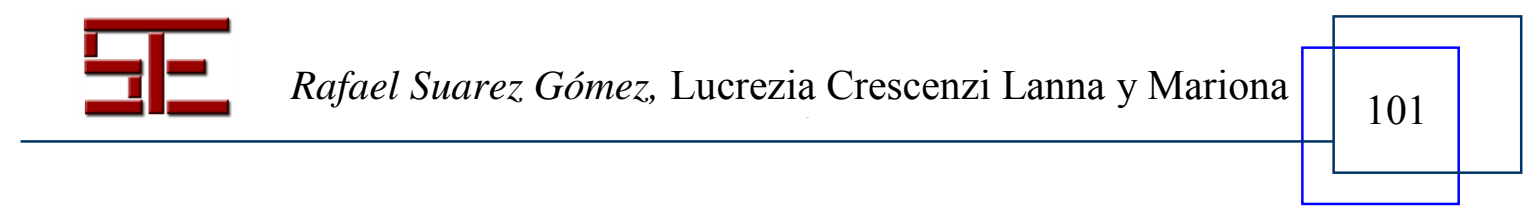




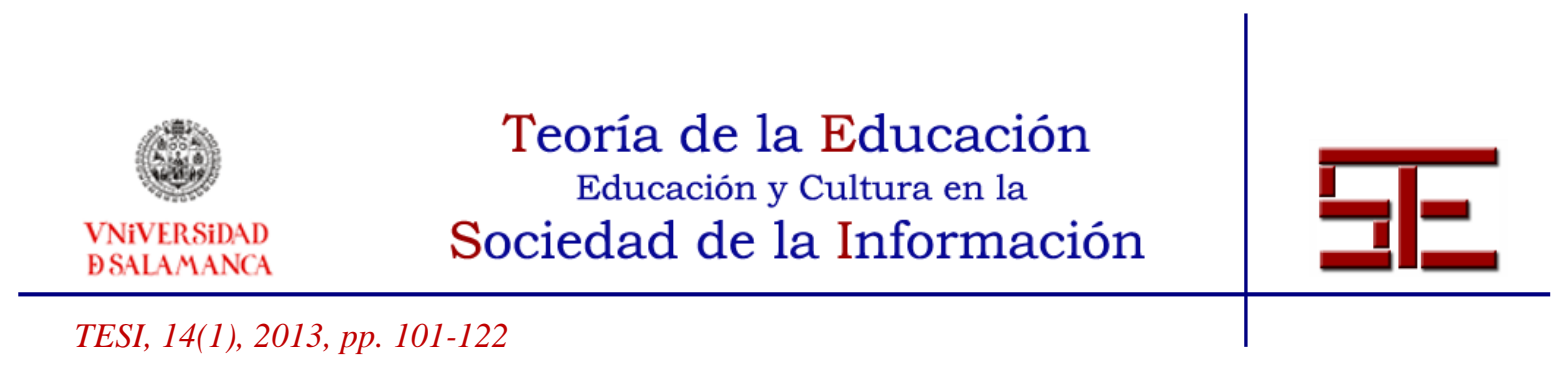

\title{
ANALYSIS OF THE COLLABORATIVE ENVIRONMENT CREATED FOR A MOBILE LEARNING EXPERIENCE
}

\begin{abstract}
We present the results of an investigation about the conceptions and uses of mobile devices in a group of students who worked on a collaborative learning environment as part of an e-learning process. We used two research methods, first analyzing the content of messages posted on a discussion forum throughout the course, to collect data on the use and evaluation the students made of the mobile learning process. Subsequently, in-depth interviews, we examined the perception of mobile learning and the changes produced during the course in the collaborative learning environment. The results indicate that the exchange environment created amplifies the participation and collaboration between students in the process of mobile learning, encouraging a greater role for students in an online learning experience.
\end{abstract}

Key words: discussion forum; online learning; collaborative environments; mobile learning; in-depth interview.

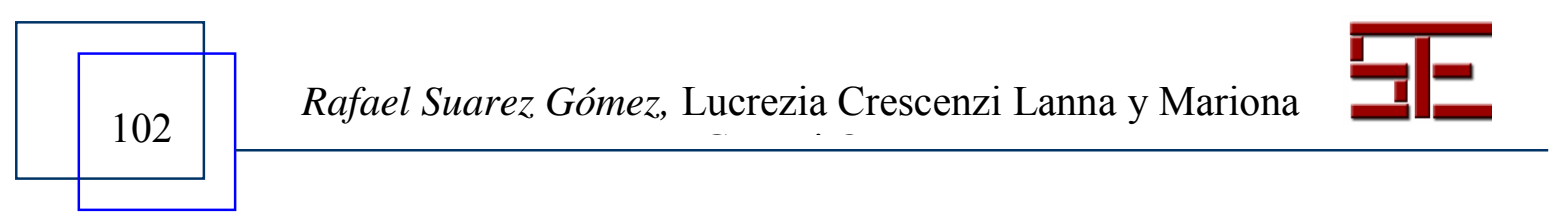




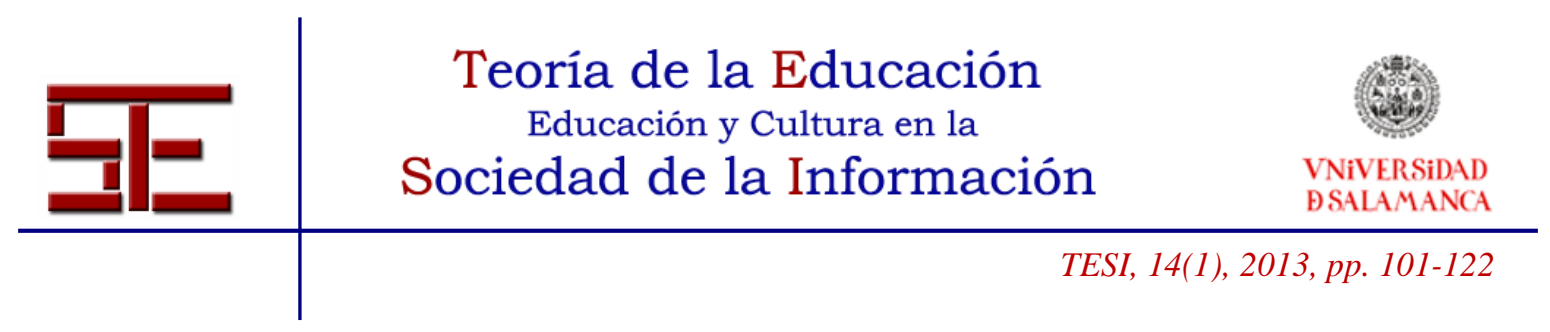

\section{ANÁLISIS DEL ENTORNO COLABORATIVO CREADO PARA UNA EXPERIENCIA DE MOBILE LEARNING}

Fecha de recepción: 10/11/2012; fecha de aceptación: 07/01/2013; fecha de publicación: 28/02/2013

Rafael Suárez Gómez

rsg2046@gmail.com

Universidat de Barcelona

Lucrezia Crescenzi Lanna

1.crescenzi@ub.edu

Universitat de Barcelona

Mariona Grané i Oro

mgrane@ub.edu

Universitat de Barcelona

\section{1.- INTRODUCCIÓN}

El presente artículo presenta resultados de una investigación realizada en colaboración con el Instituto de Formación Continua (IL3) de la Universitat de Barcelona y tiene como finalidad el análisis de las concepciones y usos de los dispositivos móviles en los procesos de aprendizaje e-learning.

Con tal objetivo, un grupo de alumnos de Postgrado del IL3 recibieron al principio del curso una Tablet PC de la compañía Apple (iPad, tal y como se denominará de aquí en adelante) como herramienta del proceso de mobile learning. El estudio se centró en el análisis de la concepción de movilidad de los estudiantes, los cambios que introducen las herramientas móviles en los procesos de aprendizaje, la asiduidad con la que la utilizan, su finalidad y las herramientas y sistemas que utilizan durante su uso.

Este estudio fue realizado durante 16 meses, iniciándose en Septiembre de 2010. Se emplearon metodologías diferentes para estudiar los usos y valoraciones de los estudiantes. Cada una de las metodologías permitía recoger datos con tal objetivo y

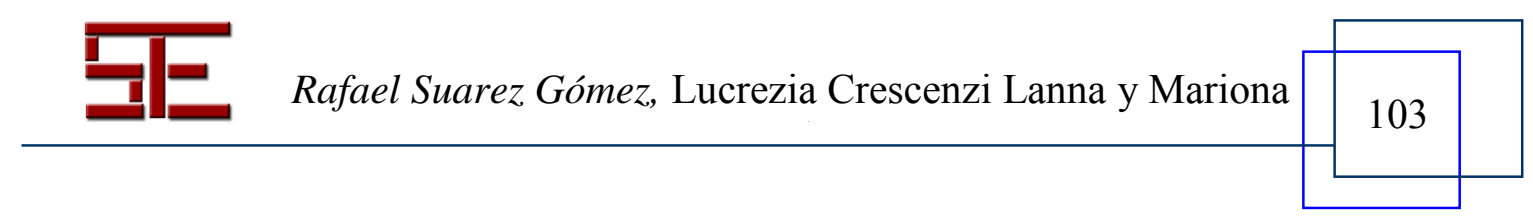




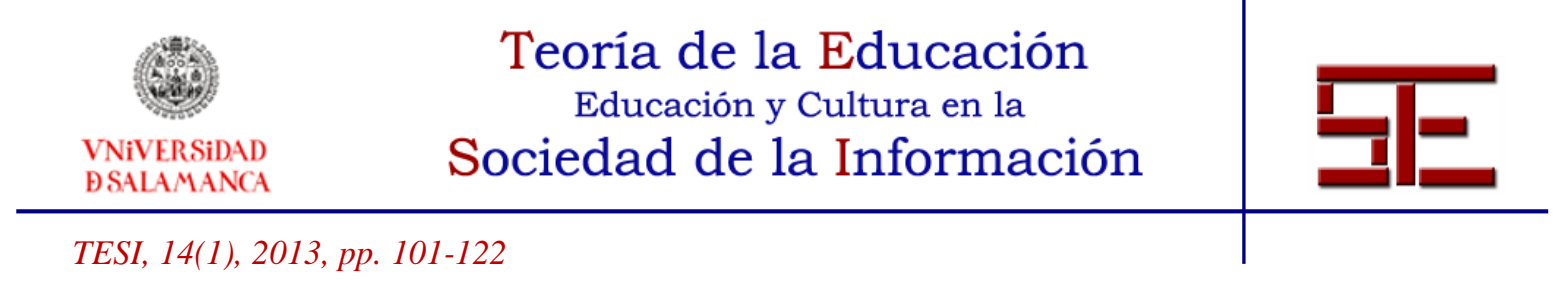

posteriormente se realizó una triangulación metodológica como "combinación de métodos de investigación" (Igartua \& Humanes, 2004). Las metodologías empleadas son:

- El análisis de contenido de los mensajes de un foro de debate: para registrar y analizar las valoraciones, sensaciones, puntos de vista, problemas y opiniones de los alumnos.

- La Entrevista en profundidad a una muestra reducida de estudiantes para estudiar no sólo sus usos y valoraciones sino sus concepciones acerca de ideas como el mobile learning.

Una de las dimensiones analizada en la investigación fue el aprendizaje colaborativo y su transformación, a partir de la experiencia de mobile learning. En particular se situó el énfasis por un lado, en las opiniones y valoraciones personales de los estudiantes, y por otro lado en el modo en que se relacionaron entre ellos, el dispositivo (iPad) y sus aplicaciones.

La investigación que se presenta, quiere ser una aportación más a los estudios sobre "mobile learning" desde una perspectiva educativa.

Sin planteamientos deterministas, aunque se considera que la tecnología actual es mucho más que una herramienta más (Gros, 2012), se parte de la comprensión de que actualmente los procesos de aprendizaje están influenciados por las posibilidades que las tecnologías de la información y la comunicación nos brindan. Posibilidades que permiten una mayor interacción del individuo, no solo con los contenidos, y los recursos de la red, sino especialmente con otros usuarios.

En este sentido es importante comprender que los dispositivos móviles, como tecnología para la comunicación y el acceso a la información, representan un cambio clave en la relación de las personas con neutro entorno: ya no nos conectamos, estamos permanentemente conectados (Bartolomé \& Grané, 2009).

Esta idea supone un poderoso cambio que tiene implicaciones más allá del hecho tecnológico como apuntan Sharples, Arnedillo-Sánchez, Milrad, y Vavoula (2009), porque se refiere a transformaciones:

- $\quad$ En el espacio físico, la ubicación de cada usuario ya no es relevante para aprender;

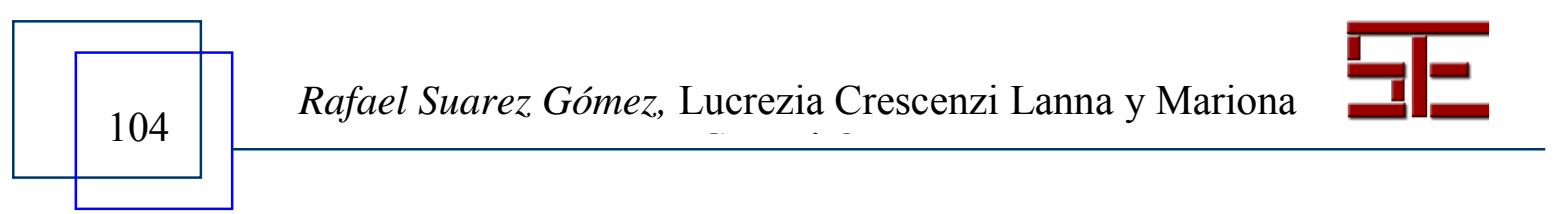




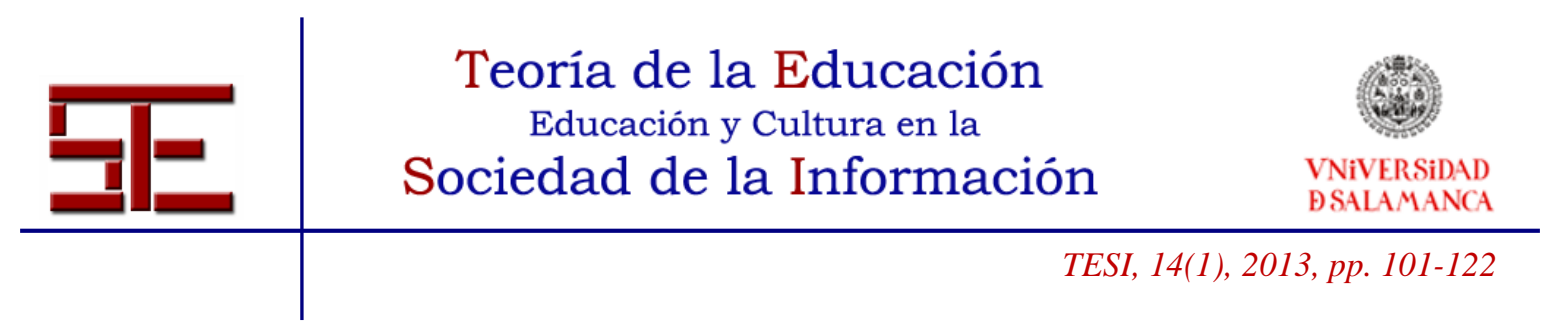

- La portabilidad en la tecnología desde las posibilidades de transferencias entre dispositivos, aplicaciones y sistemas;

- $\quad$ El espacio conceptual, en referencia a las posibilidades de poder dar respuesta a nuestras necesidades, dudas, intereses, curiosidades, compromisos...;

- $\quad$ El espacio social, que nos permite aprender, como apunta Siemens (2005) de los amigos, compañeros, conocidos... de nuestro entorno y relaciones sociales;

- El aprendizaje disperso, en el tiempo, pero también gracias a nuestras conexiones, experiencias, etc., en ámbitos de aprendizaje formales o en entornos informales donde también aprendemos.

Pero es importante comprender que estas no son solamente aportaciones de las tecnologías móviles, sino que son posibles por la propia evolución actual de las TIC. La web social y participativa (o Web 2.0 según O'Reilly, 2005), aborda directamente esta perspectiva que permite a los usuarios ser los protagonistas en la creación de contenidos, compartir ideas, construir conocimiento de forma colaborativa, establecer conexiones, desarrollar proyectos, etc. Ya en el 97 Lévy expuso que si existieran las tecnologías que permitieran mediar entre los agentes de un grupo dispuestos a colaborar, estos podrían potenciar su capacidad creativa. Las tecnologías hoy se han desarrollado para permitir esta colaboración social en la construcción de conocimiento.

Los dispositivos móviles, suponen una ampliación exponencial de las tecnologías de la web social y participativa, porque representan un aumento de la facilidad de acceso a los datos y de creación de contenidos textuales y audiovisuales, e implican una situación de conexión en cualquier momento y lugar, la ubicuidad es la clave.

Pero no es una finalidad de esta investigación comprender la tecnología móvil, sino descubrir las perspectivas, las concepciones de los usuarios de estas tecnologías, y su visión de como influyen en las propias estrategias de aprendizaje y acceso a la información. Por esta razón, el presente artículo se centra en el análisis y la valoración (desde un caso concreto) del modo de aprendizaje que se genera no sólo en entornos móviles sino también en los contextos generados a partir de ellos debido a la conexión entre personas y entornos, tal y como plantea Siemens des del conectivismo (2005).

Asimismo, la mayor visibilidad y significación del mobile learning en los procesos de aprendizaje tanto en la educación superior, como continua, sugieren que en el panorama actual existen mayores posibilidades no solo para acceder al conocimiento sino también de generar nuevas estrategias de aprendizaje (Cobo, 2009).

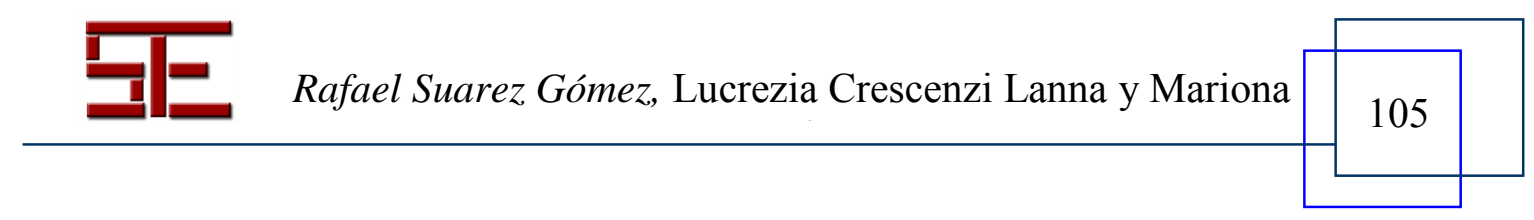




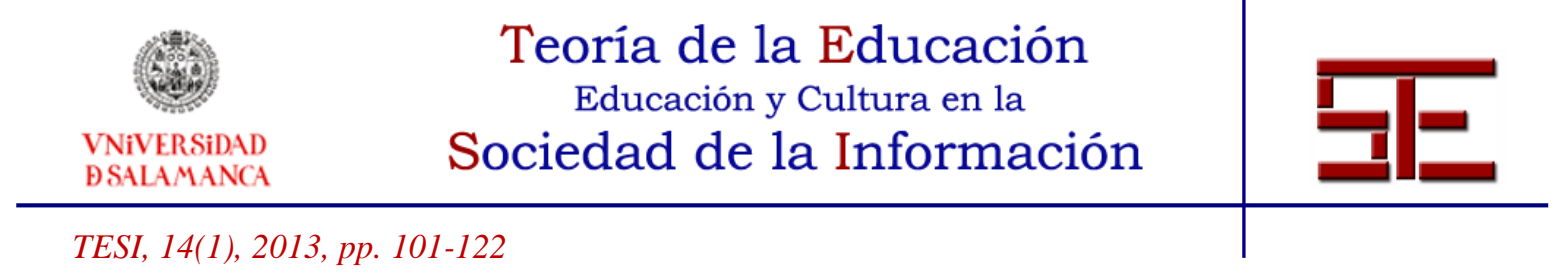

Como se utilizan, y como se quieren utilizar las tecnologías en nuestros procesos de aprendizaje es un hecho relevante en el momento actual, porque enlaza en la concepción de nuestra relación con el conocimiento y, de forma destacada, se descubre que los límites entre la vida personal y social, la vida profesional, y la vida educativa, desaparecen, porque las relaciones entre ellas son cada vez más ampliadas: estamos conectados y aprendemos en esta conexión, con el medio, los contenidos y los demás.

\section{2.- OBJETIVOS}

El objetivo de la investigación se centra en el análisis del uso de herramientas colaborativas dentro de un contexto de aprendizaje online. Mediante la observación estructurada de los mensajes de un foro de debate se analizaron las opiniones y las valoraciones que los estudiantes expresaban acerca de una herramienta concreta, el iPad, y de la experiencia de mobile learning que estaban compartiendo. Los resultados se contrastaron a través de entrevistas en profundidad a una muestra de los alumnos participantes.

Los objetivos específicos que se plantearon en la investigación fueron:

- $\quad$ Describir el uso que los estudiantes hacían del foro de intercambio.

- Analizar el uso que los estudiantes hacían de los entornos colaborativos en un entorno de aprendizaje virtual.

- Analizar los cambios que se produjeron respecto a su concepción del mobile learning en los estudiantes antes y después del proceso de aprendizaje virtual.

\section{3.- METODOLOGÍA}

Se han utilizado dos metodologías para realizar el análisis y la descripción del uso que los estudiantes han experimentado a partir de la creación del entorno colaborativo en el curso de enseñanza virtual:

- $\quad$ El análisis de contenido.

- $\quad$ La entrevista en profundidad.

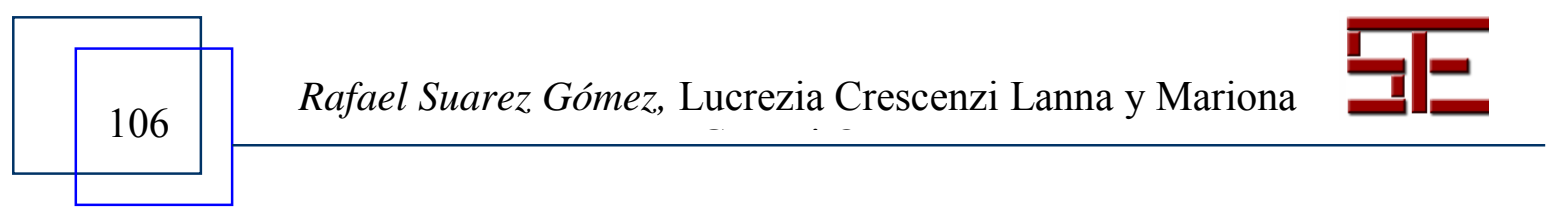




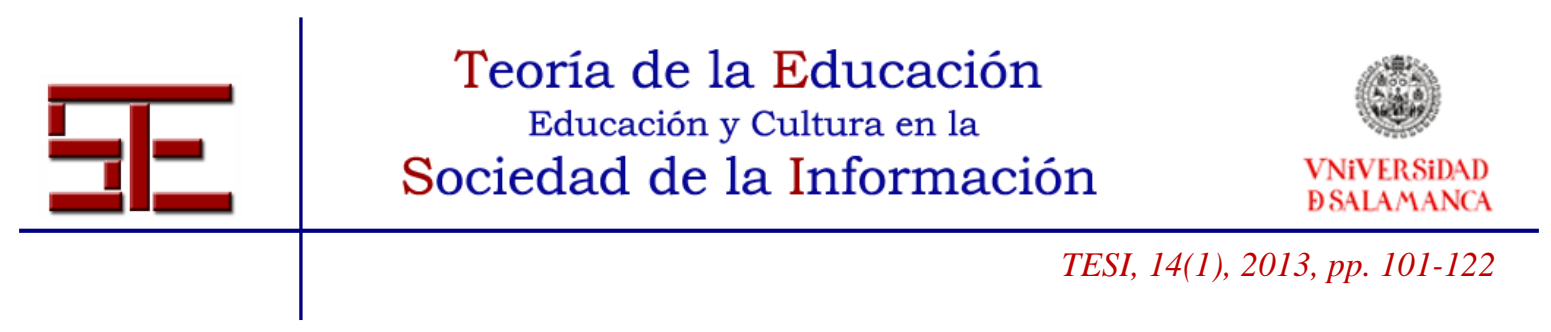

\subsection{El análisis de contenido}

El análisis del contenido se ha empleado para realizar el análisis de los mensajes creados en el foro de intercambio, un espacio donde los estudiantes podían libremente consultar dudas y aportar opiniones o valoraciones sobre la herramienta facilitada al inicio del curso. El análisis de contenido (Abela, 2003, 2):

[...] se basa en la lectura (textual o visual) como instrumento de recogida de información, lectura que a diferencia de la lectura común debe realizarse siguiendo el método científico, es decir, debe ser, sistemática, objetiva, replicable, y válida. [...] lo característico del análisis de contenido y que le distingue de otras técnicas de investigación sociológica, es que se trata de una técnica que combina intrínsecamente, y de ahí su complejidad, la observación y producción de los datos, y la interpretación o análisis de los datos.

El iPad fue entregado a los estudiantes al inicio de la experiencia de mobile learning y se realizó una observación estructurada de los mensaje durante todo el curso que empezó el mes de noviembre de 2010y terminó en mayo de 2011.

\subsubsection{El foro de debate: unidad de análisis, muestra y plantilla de observación}

Tal y como señala Martín de la Hoz (2007): “[...] el foro, como espacio de encuentro y dinamización del grupo de clase que participa de forma asíncrona en un aula virtual, aporta suficiente información y contribuciones de las personas que participan, que requiere un adecuado proceso de análisis y valoración [...]”.

Partiendo de esta definición, el uso del foro de debate como herramienta de recogida de datos permite a su vez la interacción entre los alumnos y su colaboración.

Para analizar los datos obtenidos a través de esta herramienta se creó una plantilla de observación a medida. Para realizar las observaciones se estableció la entrada como unidad de codificación. Al referirse a la entrada se entiende cada una de las intervenciones que se introduce en el foro de debate creado. El total de entradas analizadas fue de 575 .

Los alumnos que participaron en el foro fueron 72 de un total de 92 . El grupo participante está compuesto por 37 mujeres y 35 hombres, entre 25 y 55 años (34 de media). La mayoría son de nacionalidad española (86\%).

Las categorías utilizadas para la observación son el resultado de un proceso polifásico

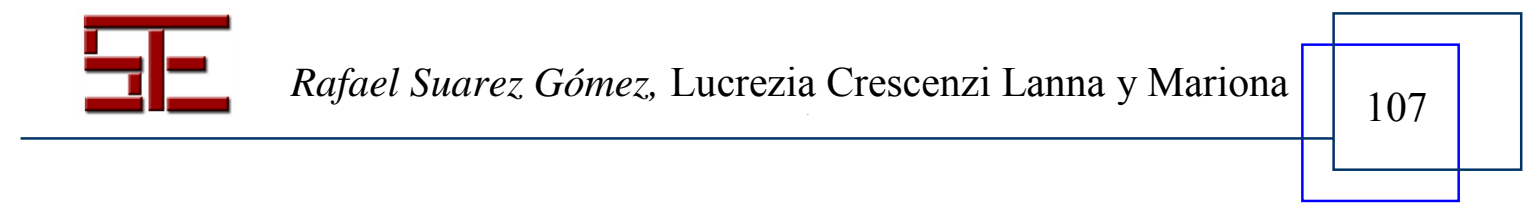




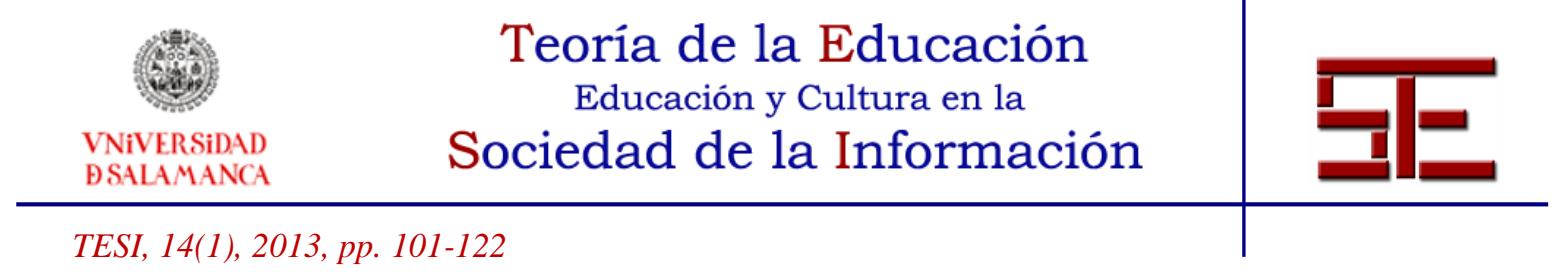

que atendía a la necesidad de que cada categoría respondiera a los criterios de exclusión mutua, exhaustividad y validez y que se basó en una adaptación del modelo de análisis de contenido empleado por Torres y Parera (2009) para el estudio de la "comunicación mediada por ordenador" (2009, p. 91).

El proceso de categorización realizado puede resumirse en cuatro etapas:

- Etapa 1: construcción o modificación del conjunto de categorías de observación y su definición.

- $\quad$ Etapa 2: observación estructurada de un número de entradas variable por parte de 2 observadores independientes.

- Etapa 3: calculo de la fiabilidad inter-codificadores antes de proceder a cambiar la plantilla de observación (si fuese necesario). Al alcanzar un porcentaje de acuerdo igual o superior al $70 \%$ las categorías eran validadas para su uso.

- $\quad$ Etapa 4: reunión del grupo de investigadores participantes en el proyecto con la finalidad de discutir los cambios a realizar (si fuese necesario).

Durante el proceso de cálculo de la fiabilidad inter-codificadores se utilizó un $20 \%$ del material utilizado para el análisis, en lugar del 10\% habitual, lo que permite tener un margen de error menor. Esta decisión se justifica también debido a que en el momento concreto en que se realizó este proceso se desconocía el tamaño de la muestra (que se determinó solo al final del curso).

El foro de debate fue dinamizado por uno de los investigadores participantes y analizado por otro de los investigadores del proyecto.

\subsection{La entrevista en profundidad}

La entrevista en profundidad utilizada como herramienta metodológica puede ser definida como "[...] una técnica de obtener información, mediante una conversación profesional con una o varias personas para un estudio analítico de investigación o para contribuir en los diagnósticos o tratamientos sociales" (Ruiz Olabuénaga, 2007, 165).

La utilización de la entrevista en profundidad a una muestra de estudiantes tenía los objetivos siguientes:

- Analizar el uso del iPad por parte de los estudiantes en el contexto de una

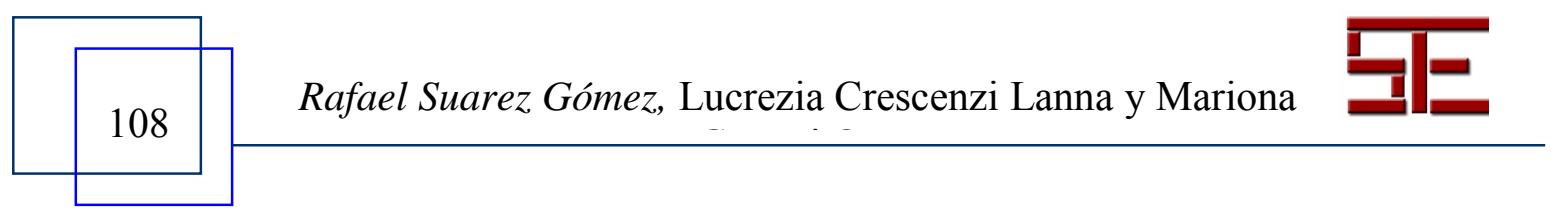




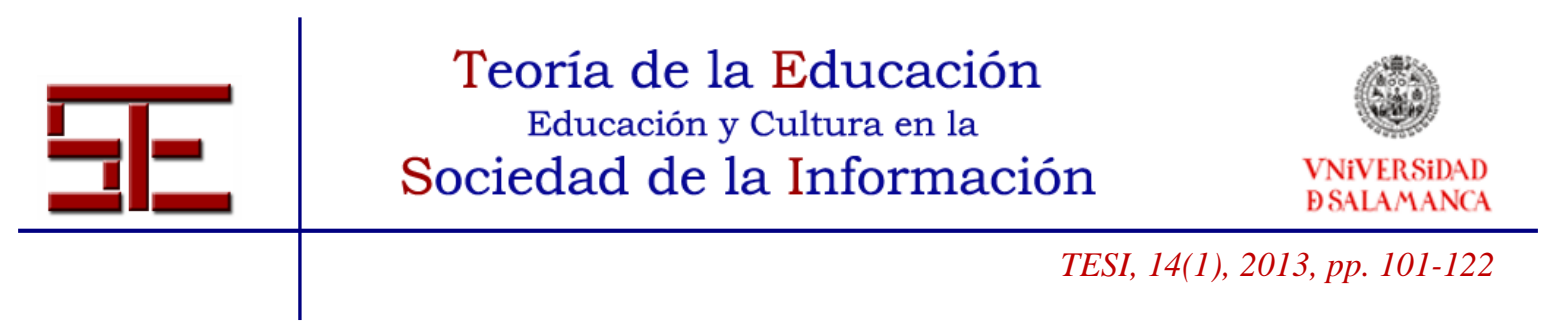

experiencia de mobile-learning.

- Analizar la valoración que hacían de su uso didáctico, profesional y personal.

- Analizar cómo integraban el uso de esta herramienta con el resto de dispositivos que utilizaban.

Por lo tanto, esta metodología permite indagar sobre la opinión de los estudiantes a partir de los resultados obtenidos a partir del análisis de contenido de los mensajes del foro de debate para así ampliar el conocimiento acerca de cómo los estudiantes entrevistados habían modificado su proceso de aprendizaje colaborativo y las vías utilizadas para hacerlo, entre ellas, el foro de intercambio.

\subsubsection{El muestreo de la entrevista}

El muestreo constó de dos partes y se compuso como sigue, con la intención de aumentar la representatividad de la muestra a pesar de su reducido tamaño:

- El $60 \%$ de la muestra se seleccionó a través de un muestreo probabilístico estratificado constante (se escogió un sujeto de cada grupo ${ }^{1}$ ).

- El 40\% de la muestra se seleccionó mediante un muestreo no probabilístico intencional teórico.

Los criterios que se consideran para esta parte de la selección de la muestra son:

(a) Uso muy frecuente VS. muy escaso de la tecnología, de Internet y de dispositivos móviles.

(b) Valoración muy positiva VS. muy negativa de tecnología, de Internet y de dispositivos móviles.

(c) Participación muy alta VS. participación mínima en el Foro.

Esta segunda técnica de muestreo es intencional teórico (y no opinático) porque los investigadores, a raíz de la experiencia y participación en el Foro de debate, seleccionan los sujetos que pueden proporcionar la mayor información posible. En el ámbito de la investigación cualitativa el procedimiento de muestreo se relaciona con la "sensibilidad" del investigador al objeto de estudio (Strauss y Corbin, 2002, 224).

Desde esta perspectiva se pidió al investigador que participó en el Forum (desde el mes de noviembre 2010) y al investigador que analizó los mensajes del mismo, que indicaran los 6 sujetos que más datos podrían aportar durante la entrevista. La selección se realizó

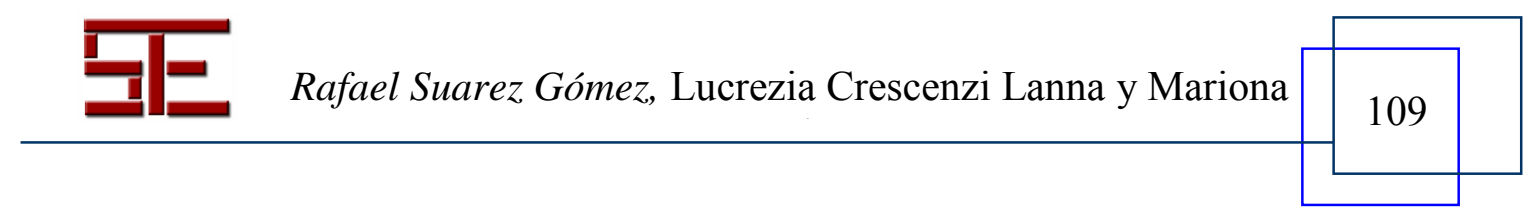




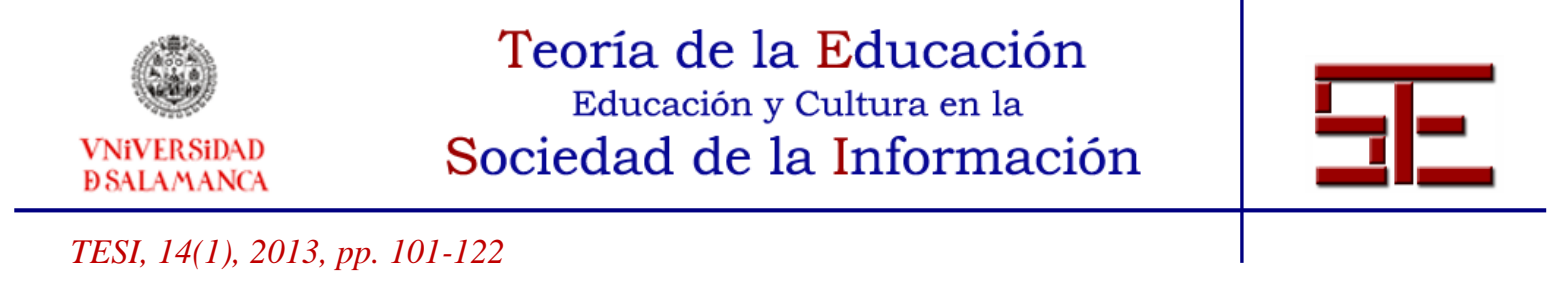

de forma independiente. Los 2 investigadores coincidieron en 4 casos sobre 6.

\subsubsection{La creación del guión}

El diseño del instrumento (guión) se validó con un grupo de expertos. Asimismo, estas categorías son las que posteriormente sirvieron para analizar los textos transcritos de las entrevistas.

Las dimensiones relacionadas con el entorno colaborativo e investigadas mediante la entrevista son:

1. Integración del iPad: se asocia con el proceso de integración del iPad en relación con otros dispositivos que los entrevistados usaran con anterioridad (teléfono móvil, vídeo consolas y portátiles, entre otros).

2. Experiencia e-learning: para conocer cómo ha sido la experiencia en un sentido amplio y general al tratarse de un curso donde se introduce un dispositivo móvil.

3. Mobile learning: con el objetivo de conocer cómo ha sido la experiencia en términos pedagógicos y didácticos específicamente.

4. Mobile life: información asociada a cómo las tecnologías móviles inciden en la vida diaria de los participantes, especialmente centrando la atención en sus actitudes, hábitos y disposición.

\subsubsection{La recogida de datos y su análisis}

En cuanto a la recogida de datos, se realizaron un total de 10 entrevistas de una duración media de 60 minutos (además de dos entrevistas realizadas como prueba piloto). Para su análisis se procedió mediante dos fases, habituales en un proceso de análisis cualitativo:

1. Transcripción completa de las entrevistas. Las entrevistas fueron registradas en formato audiovisual y se realizó su transcripción exacta. Codificación de las entrevistas: para la codificación de las entrevistas se utilizó un software CAQDAS, en concreto el programa NVivo.

Una primera aproximación a la construcción de las categorías de análisis y codificación se basó en 3 factores principales:

1. Las categorías definidas y recogidas en el proceso de construcción del instrumento

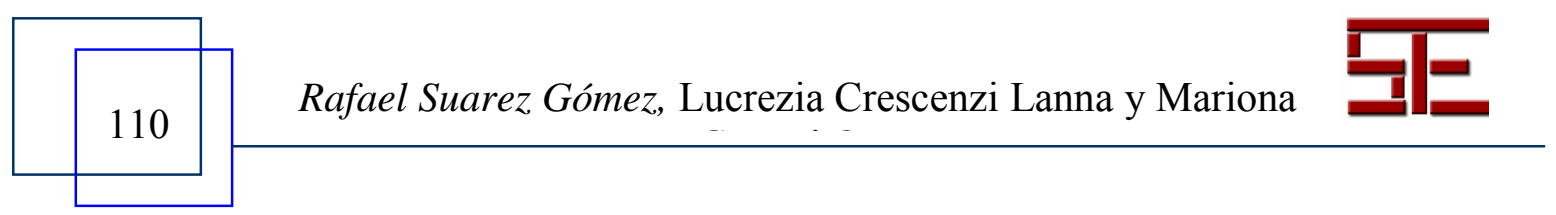




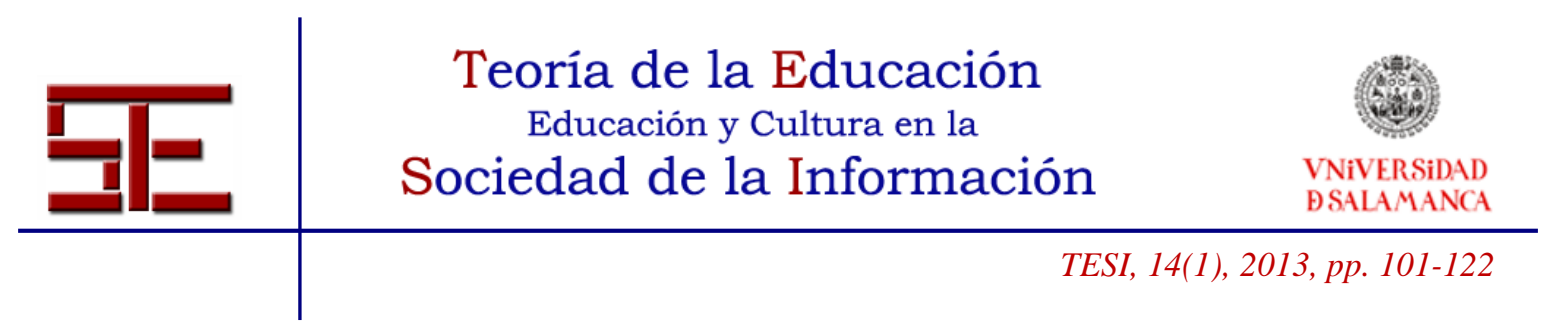

(codificación axial).

2. La participación directa en las entrevistas.

3. El proceso de transcripción.

\subsubsection{Análisis de los datos}

El análisis de los datos se realizó mediante el programa estadístico SPSS y mediante el software de análisis cualitativo NVivo. El análisis descriptivo estadístico se realizó mediante SPSS. Los datos que se analizaron mediante NVivo responden a aspectos recogidos en la entrevista a partir de la selección de palabras clave en relación a los objetivos de la investigación, los resultados de las metodologías utilizadas anteriormente y el criterio de los investigadores. Esto permite establecer la presencia y frecuencia de ciertos términos o temas, así como poder ampliar aspectos analizados de una manera más profunda a partir de respuestas personalizadas.

\section{4.- RESULTADOS}

A continuación se presentarán en primer lugar los resultados del análisis de contenido de las entradas del foro de debate. En segundo lugar, se apuntarán los resultados de la entrevista.

\subsection{Resultados del foro de debate}

\subsubsection{El foro de debate como espacio de intercambio}

Se quería investigar con qué finalidad los estudiantes empleaban el foro, observando que en el $58 \%$ de los casos a través de este espacio de intercambio los estudiantes realizaban las siguientes acciones:

1. Compartir información.

2. Exponer dudas y/o problemas.

3. Exponer soluciones.

En las demás entradas no se identificó el tipo de acción que realizaban los alumnos, por lo que fueron ignoradas para el análisis de la presente categoría.

Del $58 \%$ de las entradas donde se identificó este tipo de acción, compartir información fue la más frecuente. En el Gráfico 1 se evidencia que el $61 \%$ de las veces el foro era empleado para compartir información. Por lo tanto, el foro queda justificado como herramienta extra en un entorno de aprendizaje virtual.

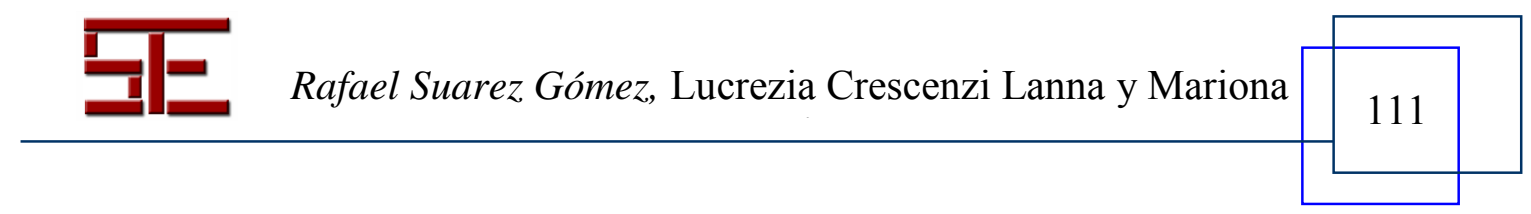




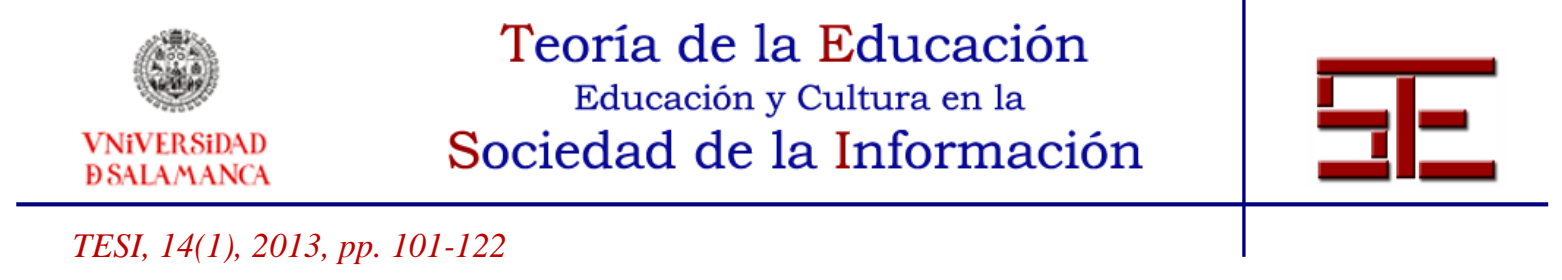

Respecto a las acciones de exponer dudas y/o problemas y exponer soluciones, los porcentajes son respectivamente del $27 \%$ y $12 \%$, lo que muestra también una predisposición a la interacción con el resto de estudiantes.

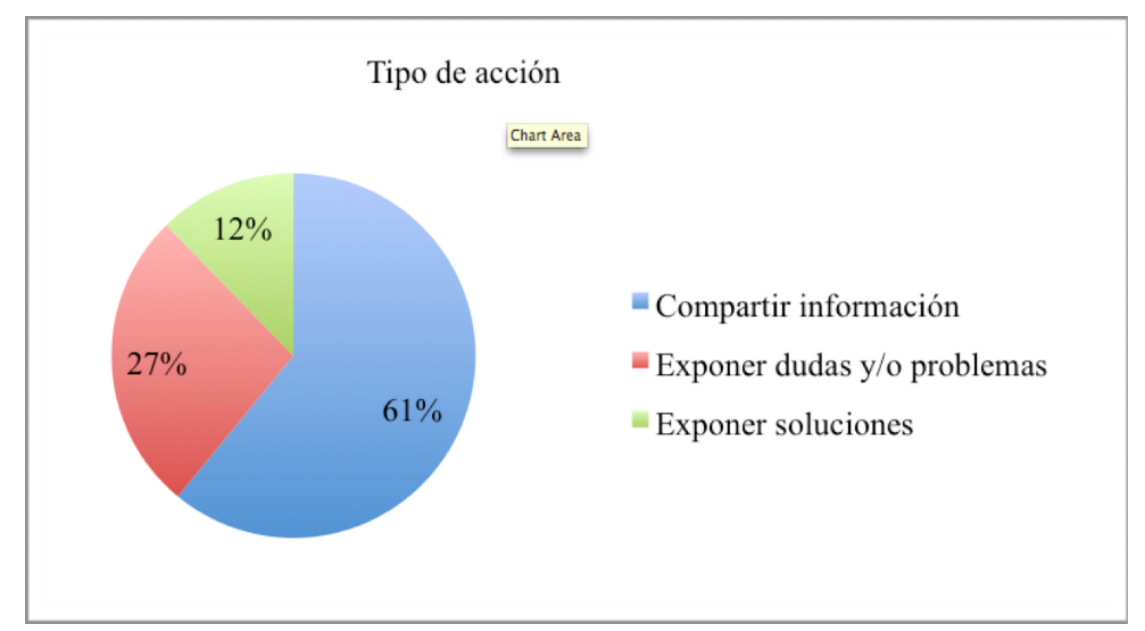

Gráfico 1. Distribución del tipo de acción identificada al utilizar el foro de debate.

Otras acciones observadas han sido la exposición de problemas encontrados tanto a nivel técnico (respecto al uso de la herramienta iPad) como académico. No obstante, no se incluyen en esta categoría sino que se analizaron en relación a otros aspectos.

Hay que señalar que la participación en el foro fue decreciendo a lo largo del curso. Tal y como se pudo comprobar mediante los resultados de la entrevista este menor uso no fue debido a la pérdida de interés sino porque los estudiantes empezaron a utilizar también nuevos entornos virtuales como X e Y para compartir e interaccionar en todo lo relativo al curso.

\subsubsection{Integración del dispositivo iPad en el curso}

Los datos recogidos indican que el dispositivo iPad fue utilizado por los estudiantes con asiduidad y que, por lo tanto, se integró al resto de dispositivos que solían utilizar con anterioridad. A la hora de realizar un curso en un entorno de aprendizaje virtual, los estudiantes valoran la posibilidad de contar con diferentes dispositivos desde los que acceder a los contenidos y actividades. En este sentido, la integración del dispositivo debe entenderse dentro de tres contextos diferentes:

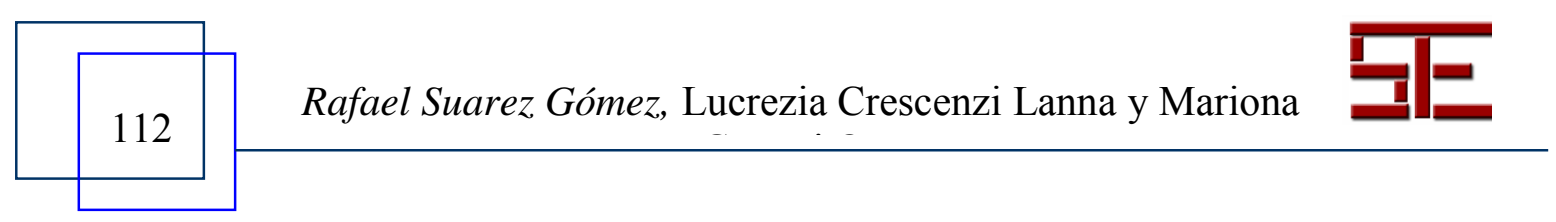




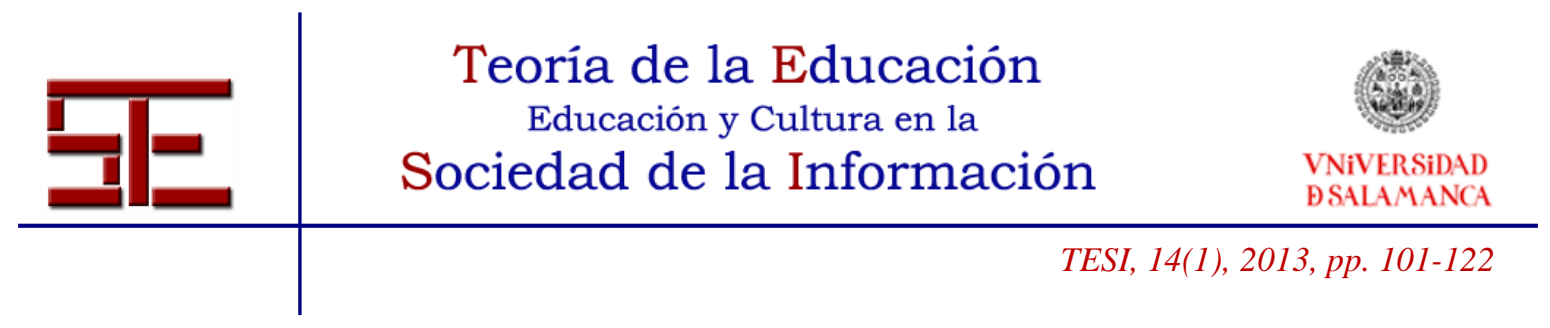

- Didáctico.

- Profesional.

- Personal.

Esta categoría (Integración) fue analizada a partir de una serie de subcategorías así definidas:

- Sustitución: se refiere a la sustitución entre dispositivos, es decir, lo que antes se realizaba con un dispositivo A, ahora se realiza con un dispositivo B.

- Complementación: se refiere a la complementación de dispositivos, utilizando unos para una finalidad y otros para diferentes fines.

- Duplicidad: se refiere al uso indiscriminado de dispositivos diferentes para las mismas acciones.

- Limitación: se refiere a la limitación que algunos dispositivos tienen para realizar acciones concretas.

- Comparación: se refiere a los comentarios donde se comparan las características de un dispositivo A con un dispositivo B.

En un total de 98 entradas (17\%) se identificó la categoría de Integración, especialmente en lo que se refiere a sus subcategorías Sustitución y Complementación. Considerando el total de las referencias a la Integración, la Sustitución en el uso de dispositivos se ha registrado en el $25 \%$ de las ocasiones y la Complementación entre dispositivos en el 30\%.
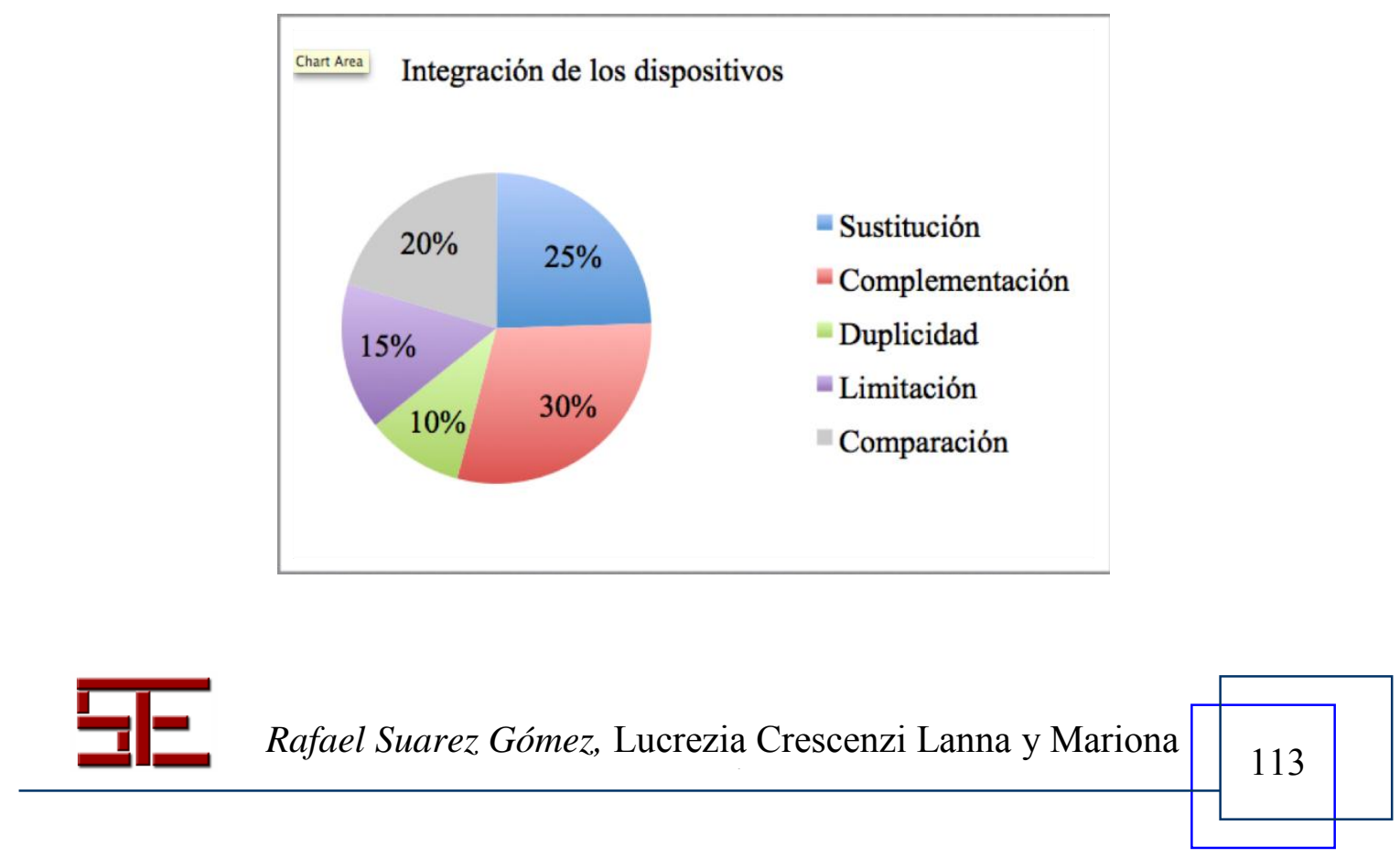


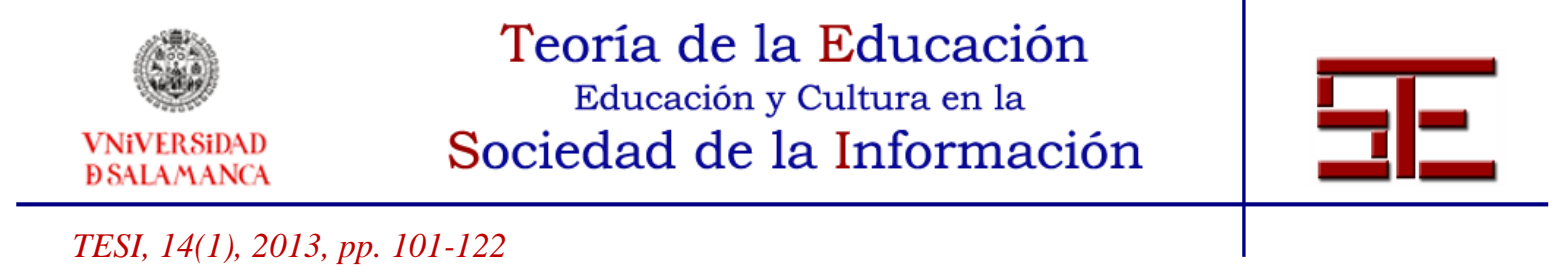

Gráfico 2. Porcentajes respecto al tipo de integración tecnológica entre dispositivos.

En este sentido, los datos de la investigación permiten indicar que la Sustitución debe comprenderse como un fenómeno contextualizado en un espacio de cambios respecto a la integración de dispositivos. En concreto, la Sustitución está relacionada con una actitud de cambio de positivo mostrada por los estudiantes y estudiada mediante estadística inferencial a partir de los resultados obtenidos mediante el análisis de contenido.

Los datos estadísticos extraídos del análisis del foro también muestran que la integración de nuevos dispositivos tecnológicos en un entorno de aprendizaje virtual permite a los estudiantes acceder a los contenidos y compartirlos a través de diferentes opciones. No necesariamente esto implica que exista un dispositivo concreto que prefieran sobre otros, pero sí que la posibilidad de trabajar con diferentes dispositivos se valora de forma positiva.

\subsection{Resultados de la entrevista en profundidad}

Los resultados de la entrevista en relación a los objetivos planteados pueden dividirse en diferentes acotaciones:

- Integración del iPad: descripción de cómo el iPad se ha integrado al resto de dispositivos que utilizaban con anterioridad.

- $\quad$ Experiencia mobile learning: descripción de su concepción del mobile learning y de su valoración de la experiencia.

- Mobile life: descripción de la inclusión del mobile en todos los aspectos de la vida diaria, más allá de los entornos de aprendizaje virtual.

\subsubsection{Integración del iPad}

Así como en el foro de discusión a través de las entrevistas se han investigado los conceptos relacionados con la integración del iPad: sustitución, complementación, duplicidad, limitación y comparación.

Las entrevistas muestran una constante presencia de comparaciones entre dispositivos, no solo con dispositivos con los que contaban antes de acceder al iPad sino también con herramientas tradicionales como el papel y el lápiz.

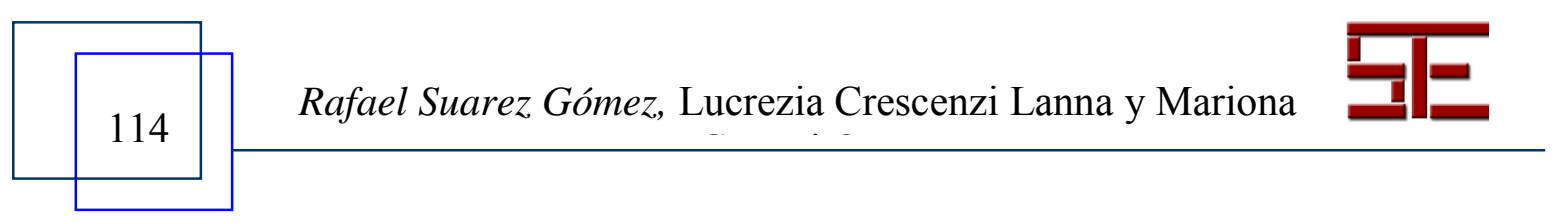




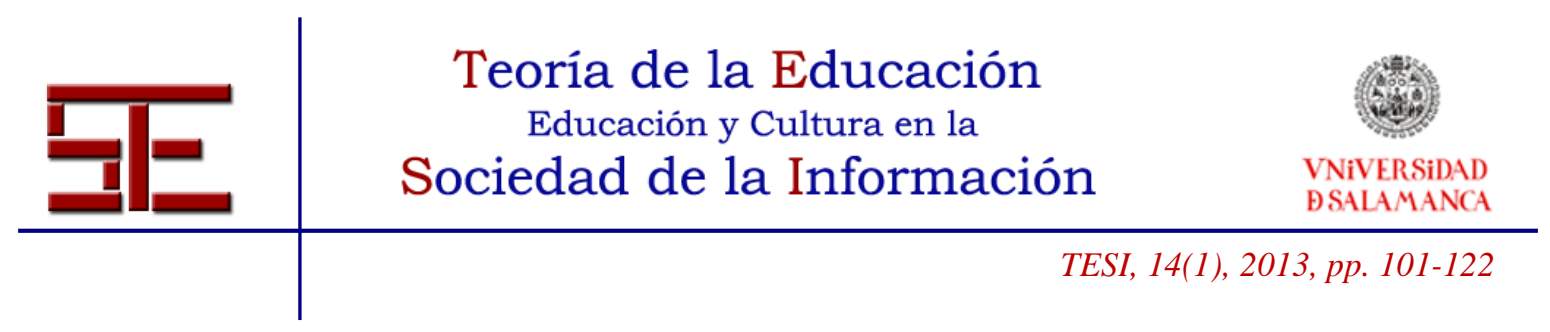

En cuanto a la combinación de dispositivos, se utilizan diferentes dispositivos en función de la tarea que desean realizar. En este sentido, el uso del iPad es valorado positivamente gracias a la movilidad que permite frente al portátil o la visibilidad de la pantalla frente a la de los teléfonos móviles smartphone.

Por ejemplo, hablando del iPad, uno de los entrevistados indica: "Sí claro, lo seguiré utilizando para leer, para consultar correo, navegar por Internet. Todo eso, es súper cómodo y no es ni un ordenador ni un portátil que lo tienes que encender y tardas más minutos y es más engorroso".

En este sentido, y en relación a la movilidad que ofrecen un iPad o un smartphone, es el ordenador el que queda relegado: "No no, el móvil no lo he dejado de utilizar, pero sí que utilizo menos el ordenador principal según lo que tenga que hacer si es nada más navegar y tal pues agarro el ipad".

No obstante, algunas de las desventajas que se indican sobre la herramienta utilizada, como su teclado o el almacenamiento de información, hacen que la combinación de los diferentes dispositivos sea indispensable a la hora de realizar un curso en un entorno de aprendizaje virtual.

\subsubsection{Experiencia mobile learning}

Considerando el mobile learning una evolución del e-learning, los estudiantes hacen referencia a su experiencia personal.

La experiencia de mobile learning es valorada positivamente también en comparación con cursos presenciales. Respecto a esto es importante evidenciar que se consideran fundamentales las tecnologías móviles al permitir estar en contacto con los compañeros y profesores en todo momento. Por ejemplo, uno de los alumnos entrevistados comenta: "Aquí la diferencia es que ahora, los trabajos ahora, los hablamos, los dialogamos, los compartimos, algunas personas los comparten... mira este es el trabajo que yo hice, la nota que saqué y el comentario del profesor. Se comparte...”.

En esta línea, uno de los aspectos más valorados ha sido el aprendizaje colaborativo y la posibilidad de intercambiar experiencias de una forma directa con el resto de los alumnos y profesores.

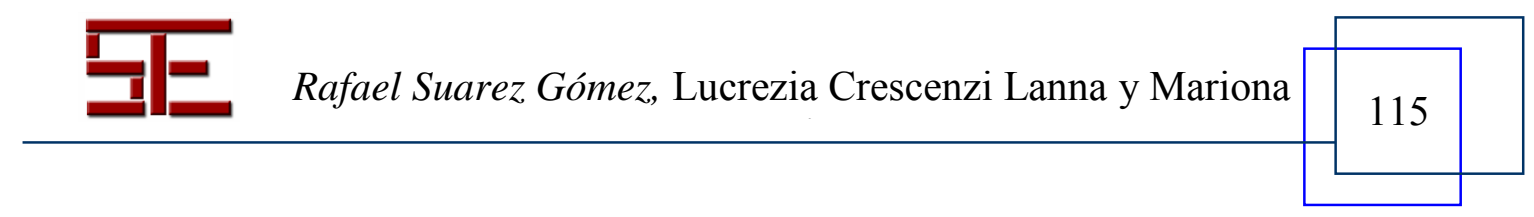




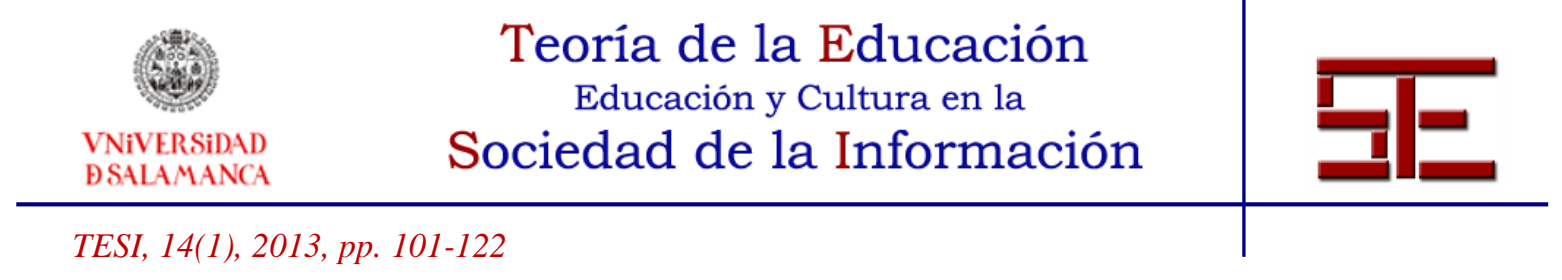

Un aspecto a resaltar son las sugerencias que alguno de los entrevistados hace acerca de las tecnologías a utilizar en los cursos online, introduciendo nuevos elementos que permitan una mayor interacción entre los participantes. Uno de los alumnos entrevistados comenta:

"Pues, las posibilidades muchas porque yo creo que se le puede aplicar ya hasta la... lo que es la realidad aumentada, esto, o sea que posibilidades tiene todas y más, desde juegos a aprendizaje mediante juegos, el game-learning o que creo que ya... no ya ... IL3 ya tiene una actividad, por lo menos aún es piloto, pero ya por esa vía, por esa vía hay muchísimas posibilidades, está en pañales.."

En otra entrevista se observa: "Me gustaría que fuera tipo second life, o sea que... que es un entorno virtual donde tu entras y... es como si nosotros estuviéramos digitalizados y de repente hay un profesor, digital, y de repente tú haces click. ¿Sabes?, como una aventura gráfica, tú haces click y puedes hablar con el profesor conversar..."

La valoración de la experiencia de mobile learning es positiva también al observar que 9 de los 10 entrevistados contemplan la posibilidad de realizar cursos de aprendizaje online en el futuro.

Los aspectos más relevantes que señalan como principales para el éxito de un curso online son:

- El feedback.

- $\quad$ La tutoría y el seguimiento adecuado de los profesores o tutores.

- $\quad$ La participación de sus compañeros y de los profesores o tutores.

Todos estos factores indican que las tecnologías influyen en el desarrollo de los cursos online y en el proceso de aprendizaje especialmente identificado en la posibilidad de interactuar, participar e intercambiar.

\subsubsection{Hacia el mobile life}

La introducción de dispositivos móviles en el aprendizaje no es una excepción. El mobile learning es una vertiente más de lo que se pueden identificar como mobile life, es decir, la introducción de las tecnologías móviles en todos los ámbitos sociales. En este sentido, los datos recogidos mediante la entrevistan indican que los alumnos, en concreto 7 de 10 , no consideran que la tecnología haya modificado sus hábitos y costumbres. No obstante,

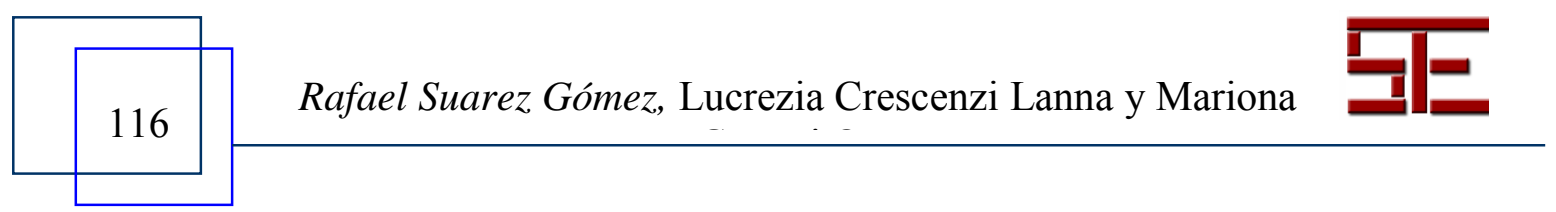




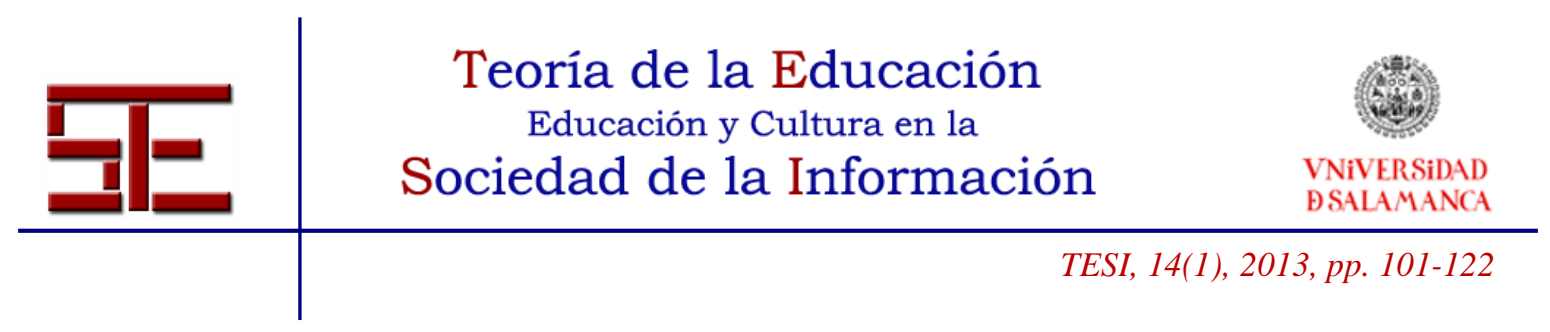

al analizar respuestas a otras preguntas, se evidencian cambios tanto en los hábitos como en las actitudes respecto a la tecnología, indicando no ser conscientes de ellos.

Por ejemplo, uno de los alumnos entrevistados, tras empezar a utilizar nuevos dispositivos móviles, indica que el hecho de estar conectado siempre es una característica de la actualidad:

\footnotetext{
"Lo que me ha quitado es tiempo, o sea, la procrastinación famosa es lo que me ha cambiado. Si antes tenía que hacer una cosa me ponía, hay que hacer esto y lo voy a hacer ahora, pues al tener cosas como esta o como el iPhone, al final haces cosas, dices... me lo estoy pasando bien, pero ¿tendrías que estar haciendo otras cosas no?"
}

También se evidencian cambios producidos por la influencia de las redes sociales, por el acceso instantáneo a la información, así como por el aumento de sus actividades en la Red ya sea para fines profesionales o personales. Uno de los entrevistados comenta: “[...] hace diez años esto no existía y todos éramos felices, ahora tienes que estar localizable para todo, para todos, y si no lo estabas, es como... ¿te pasa algo? y eso es muy curioso".

Finalmente, otros aspectos que se destacan en relación al mobile life por parte de los entrevistados son la posibilidad de esquivar las barreras geográficas para trabajar y conocer a otras personas, así como la posibilidad de ponerse en contacto con otras personas para llevar a cabo proyectos en común, valorando este aspecto de la tecnología en relación con la posibilidad de la formación no presencial.

\subsubsection{Del foro de debate a las redes sociales}

Tal y como se ha indicado anteriormente, la participación el foro de debate fue disminuyendo paulatinamente. Este factor, contrastado por el investigador que dinamizaba el foro, está ligado a los datos que se recogieron posteriormente mediante las entrevistas en profundidad, donde se pudo comprobar que los estudiantes habían pasado de participar en el foro como herramienta de intercambio a las redes sociales, creando grupos de clase donde participaban junto a los profesores y se produjo una interacción valorada muy positivamente.

Por ejemplo, uno de los entrevistados, comenta: "estamos todo el día ya te digo conectados, o al whatsapp o al facebook y nos vamos soltando mensajes".

Uno de los aspectos que resaltan del uso de las redes sociales frente al foro de debate

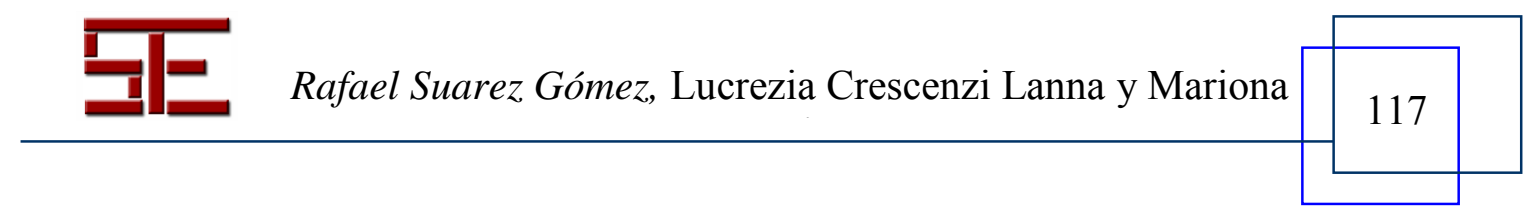




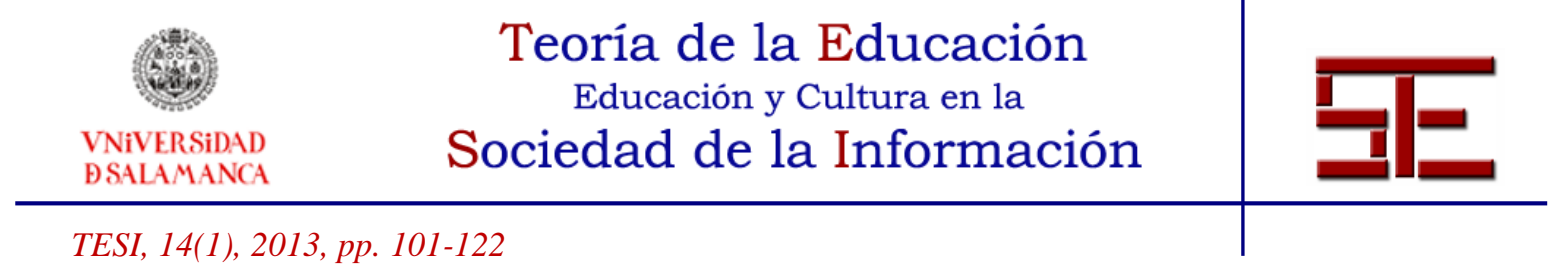

queda expuesto de la siguiente manera (en referencia al foro):

“[...] tiene que permitir las valoraciones de los alumnos. Por ejemplo, en Moodle dejamos comentarios en los foros pero no tenemos un botón de me gusta el comentario por ejemplo, no podemos valorar lo que ha dicho otro compañero. En un grupo de facebook si puedes hacerlo, entonces tiene que aportar todas las ventajas que aportan las herramientas tipo facebook, twitter y demás y combinarlas bien [...]"

En este sentido, es interesante la reflexión acerca del tipo de utilización que se puede dar a las redes sociales, apuntando hacia su valor en el campo profesional o de aprendizaje:

“[...] yo antes de facebook era mi prima no sé qué, la amiga de no sé quién se casa... todo era... en cambio ahora. o sea con el iPad, con el curso. el uso es más cognitivo profesional, o sea también social dentro del entorno de... pero que realmente es un crecimiento profesional, va más asociado hacia el crecimiento profesional y también pues una herramienta de diversión [...] la interacción te aporta muchísimo [...]"

Estos datos permiten acercarse al modo en que las redes sociales también forman parte de los entornos de aprendizaje colaborativo, sumándose a los recursos habituales del elearning. Esta tendencia se amplifica por la presencia de la tecnología móvil, que permite una mayor conectividad y genera como resultado una mayor participación e interacción entre los estudiantes y actores de proceso de aprendizaje.

\section{5.- DISCUSIÓN Y CONCLUSIONES}

El presente artículo analiza y describe una experiencia concreta realizada a partir de un proyecto de investigación financiado por el Instituto de Formación Continua de la Universitat de Barcelona (IL3). La posibilidad de acceder a un grupo de alumnos de un postgrado realizado de forma no presencial en esta entidad ha permitido analizar y describir una serie de aspectos relevantes sobre los entornos de aprendizaje colaborativo.

En primer lugar, el uso que los estudiantes han hecho del foro de debate e intercambio creado para el curso, donde los alumnos participaban no solo para consultar dudas respecto a la herramienta iPad, sino para valorar su uso y posibles utilidades, así como el propio uso que cada uno de ellos hizo de la herramienta. No obstante, en relación con los objetivos del artículo, este factor ha permitido describir cómo los estudiantes valoraban la posibilidad de compartir información gracias a esta herramienta, convirtiéndose en un elemento fundamental dentro de un entorno de aprendizaje colaborativo.

Asimismo, el uso del foro fue decreciendo para dar posteriormente a lo largo del curso

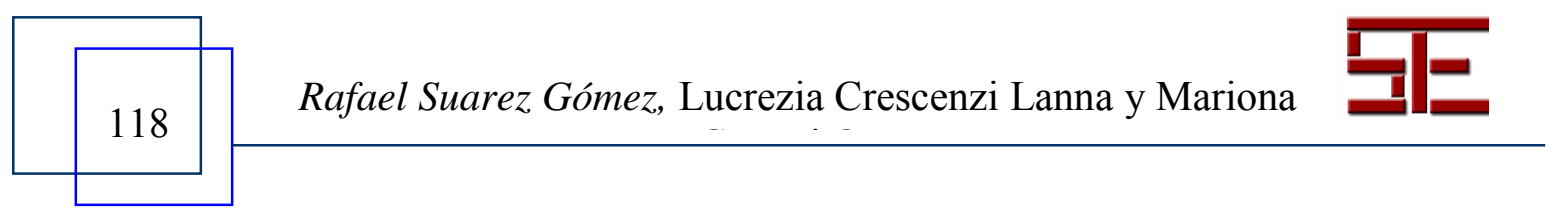




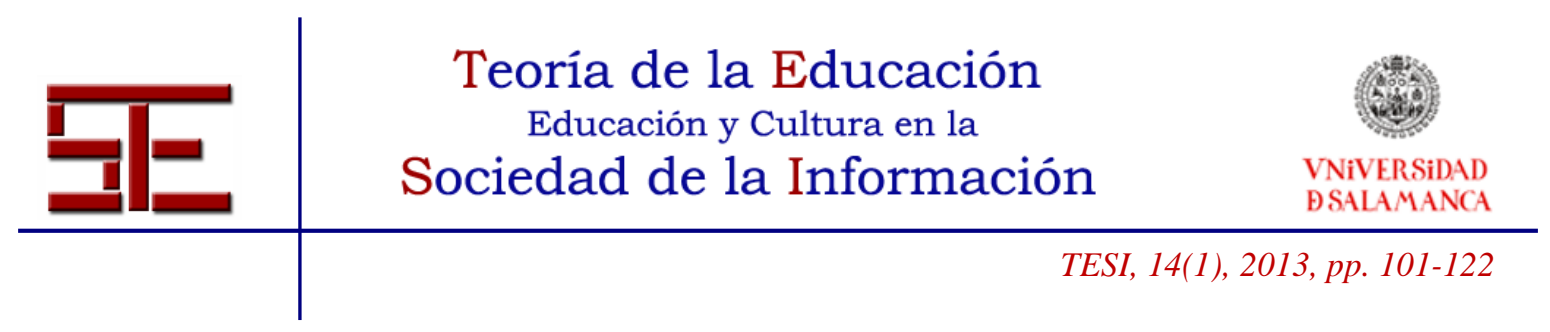

un mayor protagonismo a las redes sociales, donde los propios estudiantes crearon un grupo/clase donde trasladaron sus discusiones, debates, interacciones y actividad conjunta.

En segundo lugar, el uso que los estudiantes han dado a las herramientas colaborativas ofrecidas por el entorno de aprendizaje ha sido especialmente para compartir información. También se han expuesto dudas o problemas, así como soluciones para las mismas. Por lo tanto, las herramientas se han convertido en un espacio de intercambio e interacción que también permitía la unión entre alumnos para la realización de proyectos de manera conjunta.

En último lugar, a partir del análisis del uso de la herramienta iPad, así como de otros dispositivos móviles que los alumnos tenían en propiedad, se han descrito los cambios producidos respecto al mobile learning. Estos cambios han influido especialmente en la participación de los estudiantes, permitiendo una mayor participación e interacción desde diferentes puntos de acceso: plataforma moddle y foro de debate, móvil, iPad y ordenadores. Por esta razón, también se han considerado aspectos respecto a la integración entre diferentes dispositivos para su utilización en este tipo de entornos, llegando a las conclusiones de que los alumnos utilizan diferentes dispositivos en función del objetivo que tienen pero también que ahora los nuevos dispositivos y su movilidad les permite combinar y complementar recursos tecnológicos para realizar un curso de forma no presencial.

La utilización de diferentes dispositivos móviles en este entorno de aprendizaje también ha permitido describir la importancia de las tecnologías móviles en todos los aspectos sociales, modificando hábitos y rutinas diarias de los estudiantes y llevando la reflexión hacia un punto más allá del mobile learning situado en lo que se ha definido como mobile life.

Como conclusión final debe indicarse que la utilización de esta tecnología móvil sumada a las TIC ya conocidas es una herramienta que permite generar flujos de trabajo donde aparecen acciones de suma importancia entre los propios alumnos como la interacción continúa, una mayor participación o el feedback inmediato. Con estos elementos nos acercamos hacia una experiencia de aprendizaje de calidad donde los alumnos obtienen un mayor protagonismo al crear sus propias estrategias de trabajo y comunicación.

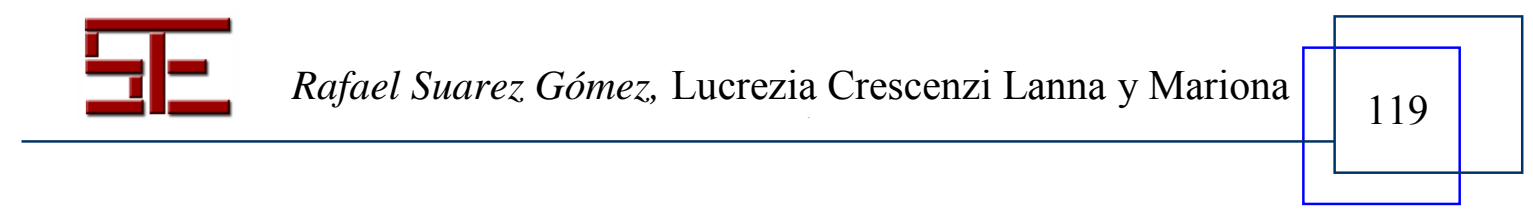




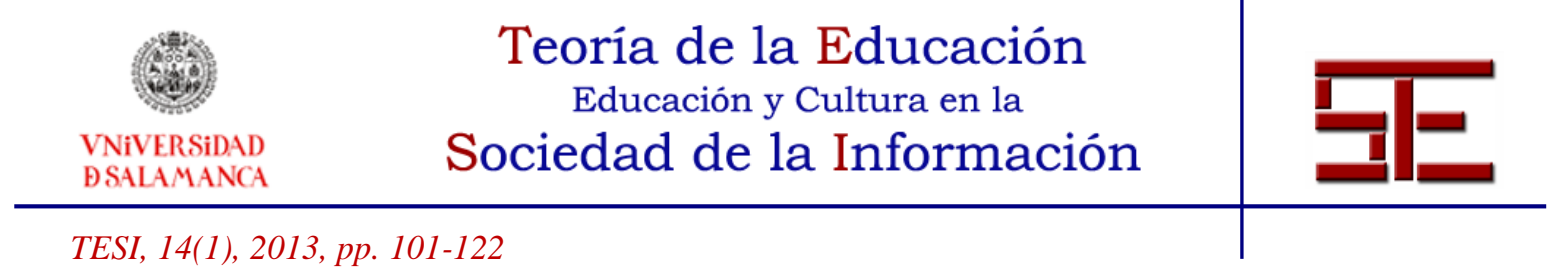

\section{6.- BIBLIOGRAFÍA}

Abela, J. A. (2003). Las técnicas de Análisis de Contenido: una revisión actualizada. Centro de estudios andaluces. Extraído el 1 noviembre, 2010, de http://public.centrodeestudiosandaluces.es/pdfs/S200103.pdf.

Bartolomé, A. \& Grané, M. (2009). Herramientas digitales en una Web ampliada. En: De Pablos, J. (Coord.). Tecnología Educativa. La formación del profesorado en la era de Internet. (pp. 351-390). Malaga: Editorial Aljibe.

Cobo, C. \& Pardo, H. (2007). Planeta Web 2.0. Inteligencia Colectiva o Medios Fast Food. México DF: Flacso México/Uvic. Extraído el 13 abril, 2010, de http://www.planetaWeb2.net/.

Gros, B. (2012). Retos y tendencias sobre el futuro de la investigación acerca del aprendizaje con tecnologías digitales. RED, Revista de Educación a Distancia. Número 32. 30 de septiembre de 2012. Extraído el 6 noviembre, 2012, de http://www.um.es/ead/red/32.

Igartua, J. J. \& Humanes, M. L. (2004). El método científico aplicado a la investigación en comunicación social. Portal de la comunicación. UAB, Aula abierta. Lecciones básicas.

Laurillard, D. (2007). Pedagogical forms of mobile learning: framing research questions. In Pachler, N. Mobile Learning towards a research agenda. (pp. 152-176). London_IOE. Extraído el 1 octubre, 2009, de http://www.wlecentre.ac.uk/cms/files/occasionalpapers/mobilelearning pachler2007.pdf

Martín de la Hoz, P. (2007). El Foro como sistema de comunicación e interacción. Revista Complutense de Educación, Vol. 18 Núm. 1 (2007) 95-112.

Olmedo, K., Grané, M. \& Crescenzi, L. (2012). Uso y percepciones de uso dispositivos móviles. Una visión desde la triangulación metodológica. Ponencia presentada en el III Congreso Internacional de la Asociación Española de Investigación de la Comunicación, AE-IC 2012 Tarragona "Comunicación y riesgo", Tarragona, España.

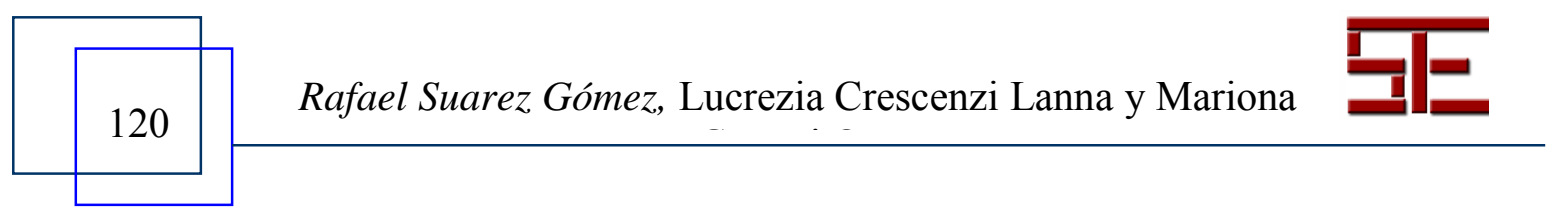




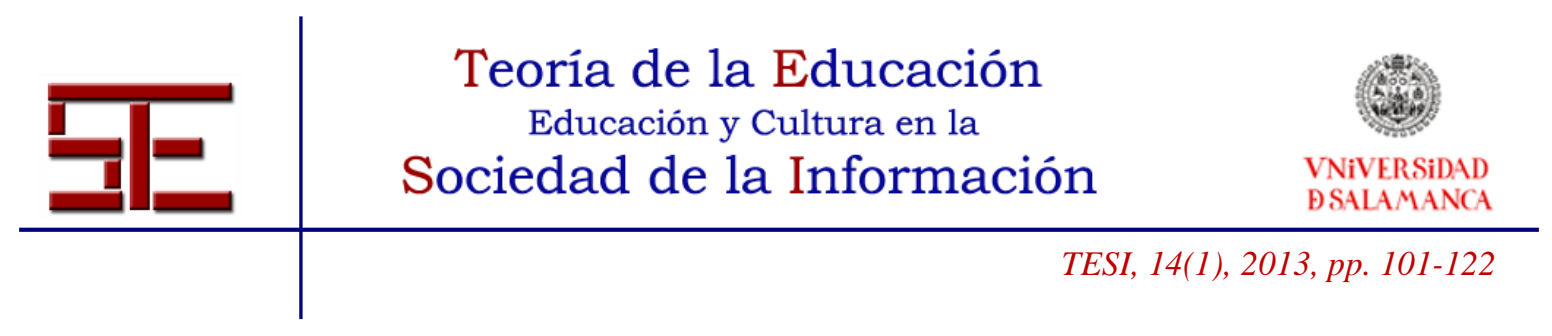

Pask, G. (1975). Conversation, cognition and learning. New York: Elsevier.

Ruiz Olabuénaga, J. I. (2007). Metodología de la investigación cualitativa. Bilbao: Universidad de Deusto.

Schank, R (2005). Lessons in Learning, e-Learning, and Training: Perspectives and Guidance for the Enlightened Trainer. Alexandria, VA: Pfeiffer.

Sharples, M., Arnedillo-Sánchez, I. Milrad, M. \& Vavoula, G. (2009). Mobile Learning. Small devices, Big Issues. In Springer (Ed.), Technology-Enhance Learning. Principles and Products. (pp. 233-249).

Siemens, G. (2005). Connectivism: A learning theory for the digital age. International Journal of Instructional Technology and Distance Learning, 2:3-10. Extraído el 2 enero, 2009 , de http://www.elearnspace.org/Articles/connectivism.htmhttp://www.diegoleal.org/social/b $\underline{\log / \text { blogs/index.php/2009/06/17/stephen-downes-el-futuro-delaprendizaje?blog=2 }}$

Strauss, A. \& Corbin, J. (2002). Bases de la investigación cualitativa. Medellín: Ed. Universidad de Antioquia.

Student Learning division of the Department of Education and Early Childhood (2011). 21 Steps to 1-to1 Success Handbook for planing, preparing, implementing and evaluating programs. Department of Education and Early Childhood: Melbourne. Extraído el 25 diciembre, 2012, de http://asp-uk.securezone.net/v2/index.jsp?id=639/684/1625

Torres Gordillo, J.J. \& Perera Rodríguez, V.H. (2009). Cálculo de la fiabilidad y concordancia entre codificadores de un sistema de categorías para el estudio del foro online en e-learning, Revista de Investigación Educativa, no 1, 2009, pp. 89-103.

Vygotsky, L. S. (1978). Mind in society. Cambridge, MA: Harvard University Press.

\section{7.- Notas}

${ }^{1}$ Los grupos se crearon a partir de los resultados de pruebas anteriores de la investigación en su conjunto (el cuestionario pre-test y el foro dirigido). Sobre este aspecto: Olmedo, K., Grané, M. \& Crescenzi, L. (2012).

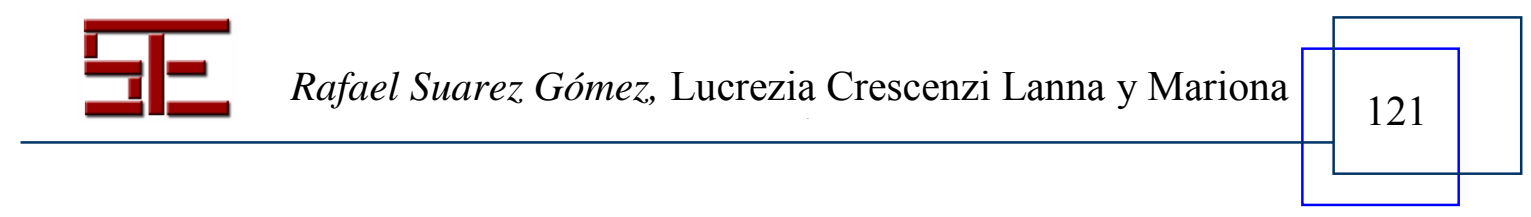




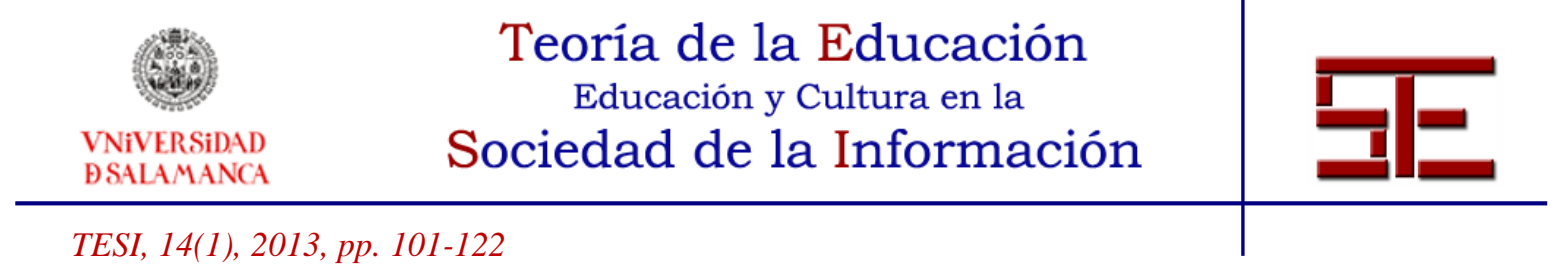

Para citar el presente artículo puede utilizar la siguiente referencia:

Suárez Gómez, R:, Crescenzi Lanna, L. y Grané i Oro, M. (2013). Análisis del entorno colaborativo creado para una experiencia de mobile learning. Revista Teoría de la Educación: Educación y Cultura en la Sociedad de la Información. 14(1), 101-121 [Fecha de consulta: dd/mm/aaaa].

http://campus.usal.es/ revistas_trabajo/index.php/revistatesi/article/view/9445/9734 